\title{
Array comparative genomic hybridization analysis in patients with anophthalmia, microphthalmia, and coloboma
}

\author{
Gordana Raca, MD, PhD ${ }^{1,2}$, Craig A. Jackson, $M S^{1}$, Laimutis Kucinskas, MD ${ }^{3}$, Berta Warman, $M S^{4}$, \\ Joseph T. C. Shieh, MD, PhD ${ }^{5}$, Adele Schneider, $M D^{6}$, Tanya M. Bardakjian, $M S^{6}$, \\ and Lisa A. Schimmenti, $M D^{4}$
}

\begin{abstract}
Purpose: The goal of our study was to determine whether genomic copy number abnormalities (deletions and duplications) affecting genes involved in eye development contributed to the etiology of anophthalmia, microphthalmia, and coloboma. Methods: The affected individuals were evaluated for the presence of deletions and duplications in genomic DNA by a very high-resolution array comparative genomic hybridization. Results: Array analysis of 32 patients detected one case with a deletion encompassing the renal-coloboma syndrome associated gene $P A X 2$. Nonpolymorphic copy number changes were also observed at several candidate chromosomal regions, including 6p12.3, 8q23.1q23.2, 13q31.3, 15q11.2q13.1, 16p13.13, and 20q13.13. Conclusions: This study identified the first patient with the typical phenotype of the renalcoloboma syndrome caused by a submicroscopic deletion of the coding region of the $P A X 2$ gene. The finding suggests that $P A X 2$ deletion testing should be performed in addition to gene sequencing as a part of molecular evaluation for the renal-coloboma syndrome. Array comparative genomic hybridization testing of 32 affected individuals showed that genomic deletions and duplications are not a common cause of nonsyndromic anophthalmia, microphthalmia, or coloboma but undoubtedly contribute to the etiology of these eye anomalies. Therefore, array comparative genomic hybridization testing represents an important and valuable addition to candidate gene sequencing in research and diagnostics of ocular birth defects. Genet Med 2011:13(5):437-442.
\end{abstract}

Key Words: array CGH, deletions, duplications, microphthalmia, anophthalmia, coloboma

$\mathrm{T}$ hree related ocular birth defects - anophthalmia, microphthalmia, and coloboma - are important contributors to childhood visual impairment and blindness, impacting 2 of 10,000 newborns annually. ${ }^{1-3}$ More than 20 different genetic loci have been implicated in congenital eye malformations, with most associated genes having a role in eye development. ${ }^{4-6}$ However, each

From the ${ }^{1}$ UW Cytogenetic Services, Wisconsin State Laboratory of Hygiene; ${ }^{2}$ Department of Pathology and Laboratory Medicine, University of WisconsinMadison, Madison, Wisconsin; ${ }^{3}$ Institute of Biology, Kaunas University of Medicine, Kaunas, Lithuania; ${ }^{4}$ Division of Genetics and Metabolism, Department of Pediatrics, University of Minnesota, Minnesota; ${ }^{5}$ Division of Medical Genetics, Department of Pediatrics, University of California, San Francisco, California; and ${ }^{6}$ Division of Genetics, Department of Pediatrics, Albert Einstein Medical Center, Philadelphia, Pennsylvania.

Gordana Raca, MD, PhD, FACMG, UW Cytogenetic Services, Wisconsin State Laboratory of Hygiene, 465 Henry Mall, Madison, WI 53706. E-mail: racago@slh.wisc.edu.

Disclosure: The authors declare no conflict of interest.

Submitted for publication July 24, 2010

Accepted for publication October 30, 2010.

Published online ahead of print January 31, 2011.

DOI: $10.1097 /$ GIM.0b013e318204cfd2 known gene is responsible for only a small percentage of cases, and many additional causative genetic factors still await identification.5,6 Mutations in the known genes account for approximately $15 \%$ of cases of anophthalmia, microphthalmia, and coloboma. ${ }^{7}$ For the remaining patients, the lack of a specific molecular diagnosis prevents prediction of long-term outcomes, anticipation of systemic complications, and estimation of the recurrence risk in their families.

Studies have shown that gene deletions and duplications may comprise up to $15 \%$ of mutations underlying monogenic disease. ${ }^{8}$ High-resolution whole genome array comparative genomic hybridization $(\mathrm{aCGH})$ testing of patients with genetic diseases can therefore detect copy number abnormalities in genes responsible for their clinical phenotypes. Application of aCGH not only detects abnormalities in known disease-causing genes but also can identify new candidate genes for specific disorders. ${ }^{8}$ Multiple examples exist of successful application of aCGH in disease gene discovery. These include implication of the TCF2 gene in the etiology of multicystic dysplastic kidneys, ${ }^{9}$ identification of PORCN as the causative gene for focal dermal hypoplasia (Goltz syndrome), ${ }^{10}$ and discovery of loci for congenital diaphragmatic hernia. ${ }^{11}$ aCGH has also contributed to identification of genes associated with congenital eye anomalies. For example, CHARGE syndrome is characterized with a specific set of birth defects, which includes coloboma, with or without microphthalmia. ${ }^{12}$ Vissers et al. ${ }^{13}$ implicated the CHD7 gene in the etiology of CHARGE syndrome by aCGH testing that detected a de novo microdeletion of the $C H D 7$ locus at $8 \mathrm{q} 12$ in an affected individual. The roles of the GDF6 gene at 8q21.2-q22.1, TFAP2A gene at $6 \mathrm{p} 24.3$, and $T M X 3$ gene at 18q22.1 in causing ocular developmental anomalies have also been discovered by testing patients who carried deletions of these genes. ${ }^{14-16}$

Although causative copy number changes have been reported in isolated cases of ocular birth defects, no one has systematically tested large numbers of affected individuals for deletions and duplications in genomic DNA. We hypothesized that ocular birth defects frequently result from copy number abnormalities involving critical genes. To test this hypothesis, we examined a cohort of patients with anophthalmia, microphthalmia, and coloboma for submicroscopic deletions and duplications by whole genome high-resolution oligo aCGH.

\section{MATERIALS AND METHODS}

Patients were enrolled through an ongoing, Institutional Review Board-approved research study "Genetics of Microphthalmia, Anophthalmia, and Coloboma" at the Division of Genetics and Metabolism, Department of Pediatrics, University of Minnesota. Written informed consent was obtained from all participants and/or their parents, as appropriate. Thirty-two patients with either isolated anophthalmia, microphthalmia, and coloboma (23 cases) or with anophthalmia, microphthalmia, and coloboma in asso- 
Table 1 Patients with syndromic and nonsyndromic anophthalmia, microphthalmia, and coloboma tested by aCGH

\begin{tabular}{lll}
\hline Sample ID & \multicolumn{1}{c}{ Ocular phenotype } & Other phenotypes (if not nonsyndromic) \\
\hline 1 & Bilateral iris coloboma & \\
2 & Bilateral optic pits & $\begin{array}{l}\text { Hypothalamic hypogonadism, mild cognitive } \\
\text { disability, hypoplastic mullerian } \\
\text { derivatives, absent ovary, left leg spasticity }\end{array}$
\end{tabular}

Previous genetic testing

Bilateral iris coloboma

$\mathrm{L}$ iris coloboma

L iris, retinal, and optic nerve coloboma

Bilateral colobomatous microphthalmia

$\mathrm{R}$ retinal coloboma, $\mathrm{L}$ microphthalmia

$\mathrm{L}$ anophthalmia, $\mathrm{R}$ microphthalmia

Bilateral colobomatous microphthalmia

Bilateral iris coloboma

Bilateral iris coloboma

Bilateral iris coloboma

Bilateral colobomatous microphthalmia, PHPV

Bilateral iris coloboma

Bilateral coloboma

Unknown coloboma, grandchildren with aniridia

Unilateral microphthalmia, morning glory

Bilateral iris coloboma

Unilateral severe microphthalmia, inferonasal coloboma, and cataract

Unilateral right complete coloboma with microphthalmia

Bilateral iris, retinal coloboma

Coloboma unspecified

Bilateral colobomatous microphthalmia

Unilateral right iris coloboma

Bilateral chorioretinal coloboma

Bilateral colobomatous microphthalmia

Coloboma unspecified

Bilateral retinal/iris coloboma

Coloboma unspecified

Right unilateral microphthalmia

Morning glory anomaly

Optic nerve hypoplasia
No information

Normal karyotype

No information

No information

No information

Developmental delay

No information

No information

UPJ obstruction and hydronephrosis

Kidney reflux

No information

No information

No information

No mutations detected in $P A X 6, S H H$, normal karyotype

Sibling of 4071

No mutations detected in PAX6, SHH, SIX6, SOX2, normal karyotype

No information

No information

No information

No previous testing

No information

No information

No previous testing

Normal Agilent 44K array, normal karyotype

No information

TORCH titres

No information

No information

Renal failure

No information

No information

No information

No information

No information

No previous testing

No previous testing 


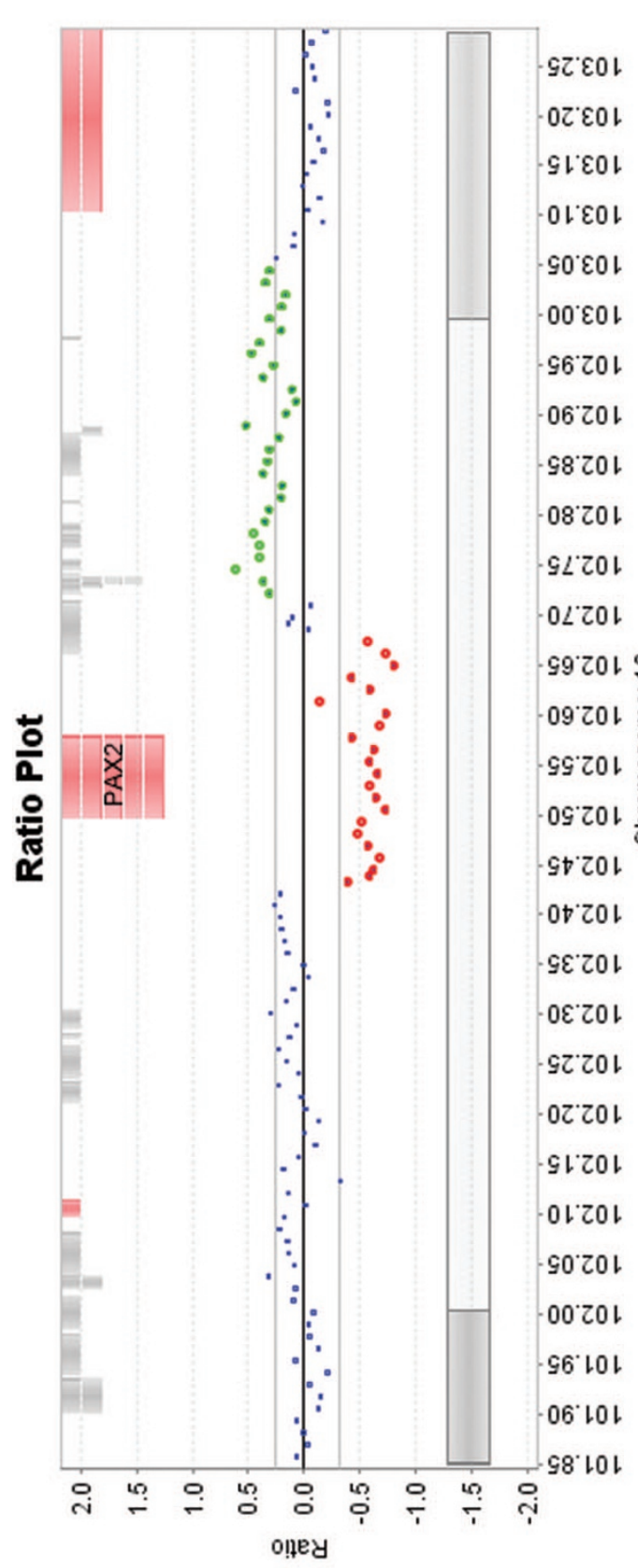

$\varangle$

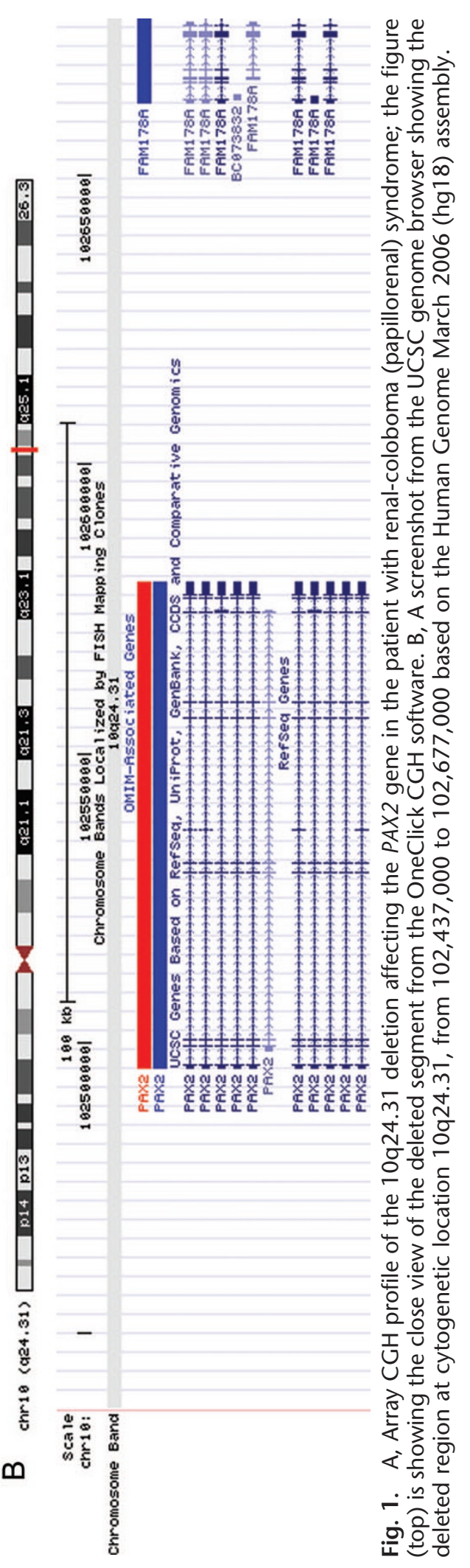


ciation with other congenital anomalies (cases 2, 6, 8, 9, 26, 28, 30,31 , and 32) were selected for testing (Table 1). All patients were evaluated by a clinical geneticist or an ophthalmologist and were enrolled in the study if they were lacking genetic diagnosis after clinical examination and standard of care testing. Molecular tests performed in each patient before the study (if known) are listed in Table 1. Array CGH analysis was performed with a commercially available HD2 human wholegenome CGH array (Roche NimbleGen Systems Inc., Madison, WI), which has approximately 2 million probes at a median interprobe distance of $1169 \mathrm{bp}$. This array can detect small genomic imbalances (deletions and duplications) at the resolution of individual genes $(\sim 5-10 \mathrm{~kb})$. Specimen labeling, array hybridization, washing, and scanning were performed at NimbleGen service laboratory in Iceland. Data analysis was performed with NimbleScan and SignalMap software from Roche NimbleGen and OneClickCGH software from Infoquant, at the UW Cytogenetic Services Laboratory at the University of Wisconsin-Madison. Regions with copy number changes detected by aCGH were compared against the database of Genomic Variants (http://projects.tcag.ca/variation/), which catalogues known benign copy number polymorphisms in the human genome. Only imbalances that do not correspond to known polymorphisms were evaluated further. Nonpolymorphic copy number changes were confirmed by separate aCGH experiments, with a different aCGH platform (EmArray Cyto6000 array Agilent Technologies, Santa Clara, CA).

\section{RESULTS}

High-resolution aCGH analysis of 32 patients with ocular defects revealed approximately $240 \mathrm{~kb}$ deletion on chromosome 10 in a patient with clinical features of renal-coloboma (papillorenal) syndrome. ${ }^{17}$ The deletion included the entire $P A X 2$ coding region and a portion of the FAM178A (C10orf6) gene (Fig. 1). This finding provided the molecular confirmation of the patient's clinical diagnosis and showed that, in addition to point mutations, deletions in the $P A X 2$ gene contribute to the etiology of the renal-coloboma syndrome.

A $5.38 \mathrm{Mb}$ duplication was detected on the long arm of chromosome 15 (cytogenetic location 15q11.2q13.1) in a patient with microphthalmia (R), anophthalmia (L), ureteropelvic junction obstruction, and hydronephrosis. The following 14 genes were affected by the duplication: ATP10A, C15orf2, GABRA5, GABRB3, GABRG3, GOLGA8E, HERC2, MAGEL2, MKRN3, NDN, OCA2, $S N R P N, S N U R F$, and UBE3A. Deletions of the same region, when inherited maternally, result in the phenotype of Prader-Willi syndrome, whereas paternal deletions of this region lead to the Angelman syndrome phenotype. Duplications of the $15 \mathrm{q} 11.2$ region, particularly when inherited on the maternal chromosome, are associated with hypotonia, autistic behavior, developmental delay, mental retardation, seizures, and mild dysmorphic features. ${ }^{18}$ However, eye anomalies have not previously been reported in patients with the $15 \mathrm{q} 11.2 \mathrm{q} 13.1$ duplications.

In addition to the deletion of the $P A X 2$ gene at $10 \mathrm{q} 24.31$ and duplication of the Prader-Willi/Angelman syndrome critical region at $15 \mathrm{q} 11.2 \mathrm{q} 13.1$, nonpolymorphic copy number changes were detected at several candidate regions including $6 \mathrm{p} 12.3,8 \mathrm{q} 23.1 \mathrm{q} 23.2$, $13 q 31.3,16 \mathrm{p} 13.13$, and 20q13.13. Based on the potential function of the genes in the regions, deletions at $13 \mathrm{q} 31.3$ and 8q23.1q23.2 were selected for further follow-up.

The $13 \mathrm{q} 31.3$ deletion was detected in a patient with bilateral iris coloboma. The deletion was approximately $240 \mathrm{~kb}$ in size and included only one gene, glypican 5 (GPC5). The GPC5 gene was a plausible positional and functional candidate gene for causing congenital eye defects in our patient; however, the $13 q 31.3$ deletion was not present in the patient's maternal first cousin who was also affected with bilateral iris coloboma (parental samples were not available).

The 8q23.1q23.2 deletion was observed in a patient with unilateral severe microphthalmia. The deletion was $1.5 \mathrm{Mb}$ in size and affected the following four known genes: PKHD1L1, $K C N V 1, E B A G 9$, and GOLSYN. Parental samples were tested by aCGH for the presence of the deletion detected in the proband, and the unaffected father was found to carry the same deletion on chromosome 8 .

A summary of all detected nonpolymorphic copy number changes including their genomic location, gene content, presence in additional family members, and likely clinical significance is provided in Table 2 .

\section{DISCUSSION}

Array CGH testing of 32 individuals with ocular birth defects detected one deletion responsible for the eye phenotype in the tested individual, one disease associated duplication that was unlikely the cause of the patient's eye anomalies, two deletions affecting strong candidate genes for eye anomalies, and three changes of completely unknown clinical significance.

The causative deletion was detected in a patient with clinical features of renal-coloboma (papillorenal) syndrome and it affected the known gene for this disorder, $P A X 2$. The deletion also included a portion of the FAM178A (C10orfo) gene, which codes for a hypothetical protein of unknown function. The partial deletion of the FAM178A gene most likely did not significantly contribute to the patient's phenotype. The patient was a 9-year-old boy with typical features of the renalcoloboma syndrome, including optic nerve hypoplasia, secondary strabismus, mild deafness, dysplastic ear helices, and renal hypoplasia. $P A X 2$ gene sequencing had been performed previously and no point mutations had been found. To our knowledge, renal-coloboma syndrome due to a deletion of the PAX2 gene has been reported in only one other patient who had a large interstitial $10 \mathrm{q}$ deletion encompassing the $P A X 2$ locus detected by high-resolution chromosome analysis. ${ }^{19}$ We report the first submicroscopic deletion affecting the coding region of the $P A X 2$ gene. No other genes likely to contribute to the patient's phenotype were affected by the rearrangement. Identification of this deletion stresses the importance of incorporating deletion/ duplication testing together with the $P A X 2$ gene sequencing into molecular diagnostics of the renal-coloboma syndrome.

Our study detected a duplication of the Prader-Willi syndrome critical region on chromosome 15 in a patient with multiple eye and kidney anomalies. Duplications of the $15 q 11.2 q 13.1$ region have been well described in the literature; when inherited on the maternal chromosome, they are associated with hypotonia, autistic behavior, developmental delay, mental retardation, seizures, and mild dysmorphic features. ${ }^{18}$ Eye anomalies have not been reported in patients with $15 \mathrm{q} 11.2 \mathrm{q} 13.1$ duplications, although a locus for the autosomal dominant colobomatous microphthalmia has been mapped to an overlapping but more distal region on chromosome $15 .{ }^{20}$ Therefore, this duplication may not be the cause of the eye anomalies in our patient, but its detection helps to explain his other clinical findings, such as developmental delay and behavioral issues.

Among the detected nonpolymorphic copy number changes, two seemed to involve likely candidate genes for eye anomalies: the $13 \mathrm{q} 31.3$ deletion and the $8 \mathrm{q} 23.1 \mathrm{q} 23.2$ deletion. Deletion of the $13 q 31.3$ region was initially considered as clinically significant based both on the chromosomal position and the function of the 





affected gene. Coloboma, microphthalmia, and anophthalmia have previously been reported in association with deletions at q31-q33 region of the long arm of chromosome 13.21 The deletion detected in our patient included the GPC5 gene, which belongs to a family of glycosylphosphatidylinositol-anchored, membrane-bound heparan sulfate proteoglycans. GPCs play a role in modulating the activity of heparan sulfate-binding growth factors. ${ }^{22}$ Their involvement in developmental morphogenesis and growth regulation has been shown by Drosophila mutants, and human genetic disorders such as Simpson-Golabi-Behmel syndrome ${ }^{23}$ and autosomal-recessive omodysplasia. ${ }^{24}$ Dally, drosophila ortholog of the gene deleted in our patient GPC5, is known to affect cell division patterning in developing eye. ${ }^{22}$ The GPC5 gene was therefore considered a plausible candidate gene for causing the congenital eye defects in our patient, but testing additional family members did not detect the 13q31.3 deletion in the patient's cousin with the identical ocular defect. Because it does not segregate with the eye anomalies, the deletion is unlikely the cause of the ocular defects in this family. However, this deletion has not been reported as a benign variant (http://projects.tcag.ca/variation/) and has been detected by another laboratory in two unrelated individuals with developmental delay and cognitive impairment (personal communication). Therefore, the clinical significance of the 13q31.1 deletion requires further investigation.

The 8q23.1q23.2 deletion was observed in a patient with unilateral severe microphthalmia and small inferonasal coloboma. He also had a dense posteriorly subluxated crystalline lens and Persistent Hyperplastic Primary Vitreous. His head magnetic resonance imaging and development have been normal. The deletion affected four known genes, including GOLSYN, which is known to play an important role in neuronal development. The unaffected father was found to carry the same deletion. It is possible that the detected deletion has incomplete penetrance and that some of the carriers do not express abnormal phenotype. Alternatively, cases have been reported where benign copy number variants contribute to abnormal phenotypes by unmasking mutations in nondeleted alleles. ${ }^{25,26}$

The 13q31.1 and 8q23.1q23.2 deletion cases illustrate difficulties in interpreting clinical significance of copy number abnormalities detected by high-resolution aCGH testing. For example, the causative role cannot be assumed solely based on the function of the affected genes. Although plausible candidate genes mapped within both deleted regions, family studies reduced the possibility of the role of the $13 \mathrm{q} 31.1$ and $8 \mathrm{q} 23.1 \mathrm{q} 23.2$ deletions in causing eye anomalies in the probands. These cases demonstrate the value of having clinical information and DNA samples available from patients' parents and other affected and unaffected members of their families.

Our study showed that aCGH could detect deletions and duplications associated with ocular birth defects. However, copy number abnormalities did not seem to be a common cause of isolated anophthalmia, microphthalmia, and coloboma. Although gene deletions and duplications significantly contribute to pathogenesis of genetic disorders, the majority of disease-causing mutations are nucleotide changes in genomic DNA. We propose that a combination of aCGH analysis with high-throughput sequencing methods that allow detection of base changes (point mutations) in a large number of candidate genes for eye malformations will be the most successful strategy for identification of new genetic causes of anophthalmia, microphthalmia, and coloboma.

\section{ACKNOWLEDGMENTS}

This work was supported, in part, by the University of Wisconsin Institute for Clinical and Translational Research through an
NIH Clinical and Translational Science Award, Grant number 1UL1RR025011, and by funding from the University of Minnesota, Department of Pediatrics. The authors thank the patients, families, and clinicians who have submitted clinical samples for the microphthalmia, anophthalmia, and coloboma study.

\section{REFERENCES}

1. Källén B, Robert E, Harris J. The descriptive epidemiology of anophthalmia and microphthalmia. Int J Epidemiol 1996;25:1009-1016.

2. Morrison D, FitzPatrick D, Hanson I, et al. National study of microphthalmia, anophthalmia, and coloboma (MAC) in Scotland: investigation of genetic aetiology. J Med Genet 2002;39:16-22.

3. Stoll C, Alembik Y, Dott B, Roth MP. Congenital eye malformations in 212,479 consecutive births. Ann Genet 1997;40:122-128

4. Gregory-Evans CY, Williams MJ, Halford S, Gregory-Evans K. Ocular coloboma: a reassessment in the age of molecular neuroscience. J Med Genet 2004;41:881-891.

5. Fitzpatrick DR, van Heyningen V. Developmental eye disorders. Curr Opin Genet Dev 2005;15:348-353.

6. Chang L, Blain D, Bertuzzi S, Brooks BP. Uveal coloboma: clinical and basic science update. Curr Opin Ophthalmol 2006;17:447-470.

7. Gonzalez-Rodriguez J, Pelcastre EL, Tovilla-Canales JL, et al. Mutational screening of CHX10, GDF6, OTX2, RAX and SOX2 genes in 50 unrelated microphthalmia-anophthalmia-coloboma (MAC) spectrum cases. $\mathrm{Br} J$ Ophthalmol 2010;94:1100-1104.

8. Vissers LE, Veltman JA, van Kessel AG, Brunner HG. Identification of disease genes by whole genome CGH arrays. Hum Mol Genet 2005;14:R215-R223.

9. Mefford HC, Clauin S, Sharp AJ, et al. Recurrent reciprocal genomic rearrangements of $17 \mathrm{q} 12$ are associated with renal disease, diabetes, and epilepsy. Am J Hum Genet 2007;81:1057-1069.

10. Wang X, Reid Sutton V, Omar Peraza-Llanes J, et al. Mutations in X-linked PORCN, a putative regulator of Wnt signaling, cause focal dermal hypoplasia. Nat Genet 2007;39:836-838.

11. Zayed H, Chao R, Moshrefi A, et al. A maternally inherited chromosome $18 \mathrm{q} 22.1$ deletion in a male with late-presenting diaphragmatic hernia and microphthalmia-evaluation of DSEL as a candidate gene for the diaphragmatic defect. Am J Med Genet A 2010;152A:916-923.

12. Sanlaville D, Verloes A. CHARGE syndrome: an update. Eur J Hum Genet 2007; 15:389-399.

13. Vissers LE, van Ravenswaaij CM, Admiraal R, et al. Mutations in a new member of the chromodomain gene family cause CHARGE syndrome. Nat Genet 2004:36:955-957.

14. Asai-Coakwell M, French CR, Berry KM, et al. GDF6, a novel locus for a spectrum of ocular developmental anomalies. Am J Hum Genet 2007;80:306-315.

15. Milunsky JM, Maher TA, Zhao G, et al. TFAP2A mutations result in branchio-oculo-facial syndrome. Am J Hum Genet 2008;82:1171-1177.

16. Chao R, Nevin L, Agarwal P, et al. A male with unilateral microphthalmia reveals a role for TMX3 in eye development. PloS ONE 2010;5:e10565.

17. Eccles MR, Schimmenti LA. Renal-coloboma syndrome: a multi-system developmental disorder caused by PAX2 mutations. Clin Genet 1999;56:1-9.

18. Browne CE, Dennis NR, Maher E, et al. Inherited interstitial duplications of proximal 15q: genotype-phenotype correlations. Am J Hum Genet 1997;61: 1342-1352.

19. Benetti E, Artifoni L, Salviati L, et al. Renal hypoplasia without optic coloboma associated with PAX2 gene deletion. Nephrol Dial Transplant 2007;22:2076-2078.

20. Michon L, Morlé L, Bozon M, et al. Physical and transcript map of the autosomal dominant colobomatous microphthalmia locus on chromosome 15q12q15 and refinement to a $4.4 \mathrm{Mb}$ region. Eur J Hum Genet 2004;12:574-578.

21. Lansink PJ, Moll AC, Imhof SM, Schouten-van Meeteren AY, Goverts ST. Variable expression of ophthalmological findings in the $13 \mathrm{q}$ deletion syndrome. Arch Ophthalmol 2005;123:127-128.

22. Filmus J, Selleck SB. Glypicans: proteoglycans with a surprise. J Clin Invest 2001;108:497-501.

23. DeBaun MR, Ess J, Saunders S. Simpson Golabi Behmel syndrome: progress toward understanding the molecular basis for overgrowth, malformation, and cancer predisposition. Mol Genet Metab 2001;72:279-286.

24. Campos-Xavier AB, Martinet D, Bateman J, et al. Mutations in the heparansulfate proteoglycan glypican 6 (GPC6) impair endochondral ossification and cause recessive omodysplasia. Am J Hum Genet 2009;84:760-770.

25. Flipsen-ten Berg K, van Hasselt PM, Eleveld MJ, et al. Unmasking of a hemizygous WFS1 gene mutation by a chromosome $4 \mathrm{p}$ deletion of $8.3 \mathrm{Mb}$ in a patient with Wolf-Hirschhorn syndrome. Eur J Hum Genet 2007;15:1132-1138.

26. Coman DJ, Gardner RJ. Deletions that reveal recessive genes. Eur J Hum Genet 2007;15:1103-1104 